\begin{tabular}{|l|c|c|c|r|}
\hline $\begin{array}{l}\text { Cuadernos de Investigación Geográfica } \\
\text { Geographical Research Letters }\end{array}$ & 2018 & N $^{\circ} 44(2)$ & pp. 641-658 & $\begin{array}{r}\text { ISSN 0211-6820 } \\
\text { eISSN 1697-9540 }\end{array}$ \\
\hline
\end{tabular}

DOI: http://doi.org/10.18172/cig.3256

(C) Universidad de La Rioja

\title{
EFECTOS DE LAS EXTRACCIONES DE ÁRIDOS SOBRE EL TRANSPORTE DE SEDIMENTOS EN SUSPENSIÓN EN RÍOS DE MONTAÑA (ALTO RÍO CINCA, PIRINEO CENTRAL)
}

\author{
M. BÉJAR ${ }^{1 *}$, D. VERICAT ${ }^{1,2}$, I. NOGALES ${ }^{1}$, \\ F. GALLART ${ }^{3}$, R.J. BATALLA ${ }^{1,4,5}$
}

\begin{abstract}
${ }^{1}$ Fluvial Dynamics Research Group (RIUS), Universitat de Lleida, Lleida, España. ${ }^{2}$ Centre Tecnològic Forestal de Catalunya, Solsona, España. ${ }^{3}$ Instituto de Diagnóstico Ambiental y Estudios del Agua, CSIC, Barcelona, España. ${ }^{4}$ Institut Català de Recerca de l'Aigua, Girona, España. ${ }^{5}$ Facultad de Ciencias Forestales y Recursos Naturales, Universidad Austral de Chile, Valdivia, Chile.
\end{abstract}

\begin{abstract}
RESUMEN. Este artículo analiza los efectos de una extracción de áridos sobre el transporte de sedimento en suspensión en un tramo del alto río Cinca (Pirineo Central). Para ello se monitorizaron cinco secciones a lo largo de un tramo de 5 kilómetros para obtener datos de caudal y transporte de sedimentos así como las granulometrías de los sedimentos finos. De manera específica, se han obtenido muestras en una sección de referencia aguas arriba del área de actuación y cuatro secciones, a 0, 200, 500 y 1500 m, aguas abajo de ésta. Los resultados confirman un efecto de las extracciones de áridos sobre el transporte de sólidos en suspensión, alcanzándose concentraciones aguas abajo de la extracción un orden de magnitud mayor que en la sección de referencia. Las $C_{S S}$ medias obtenidas durante los días de estudio son similares a los valores registrados durante crecidas, llegándose a alcanzar concentraciones máximas de casi $6 \mathrm{~g} / \mathrm{l}$. La carga sólida varía entre 2,2 y $17 \mathrm{Mg} /$ día entre secciones, valores que incorporan también los efectos de los trabajos realizados en el cauce durante los días previos a la extracción. Las concentraciones observadas en la sección más alejada son similares a las medidas en la sección de referencia, hecho que indica que la mayor parte del material movilizado por la extracción quedó sedimentado en los primeros centenares de metros. El tamaño de las partículas del sedimento removilizado y transportado como consecuencia de la extracción es mayor que el que se observa en condiciones naturales; no obstante, las partículas de mayor tamaño sedimentaron rápidamente, hecho que sugiere un lavado selectivo de los materiales transportados aguas abajo. Este estudio supone un primer paso para la comprensión de la dinámica del sedimento en ríos localmente afectados por actuaciones en el cauce y su influencia en la dinámica morfosedimentaria y la integridad bio-física del río.
\end{abstract}




\title{
Effects of gravel mining on suspended sediment transport in mountain rivers (Upper River Cinca, Central Pyrenees)
}

\begin{abstract}
This paper examines the effects of gravel mining on the suspended sediment transport in the Upper River Cinca (Central Pyrenees). Discharge, sediment transport and sediment grain-size distribution were measured, sampled and further determined in five monitoring sections along a $5 \mathrm{~km}$ river reach. Samples were taken at a section upstream from the mined area (i.e. reference section) and in four sections downstream (0,200, 500 and $1500 \mathrm{~m})$. The results show that sediment concentrations downstream from the target site were up to one order of magnitude higher than in the reference section. Average concentrations during the impact were similar to those observed during floods, with maximum values attaining $6 \mathrm{~g} / \mathrm{l}$. Total load ranged from 2.2 to $17 \mathrm{Mg} /$ day between sections; these values include the effects of the earth-moving works performed before the mining started. Concentrations at the lowermost section of the study reach were similar to those observed at the upstream reference section, suggesting that most of the suspended material was deposited in the channel. The transported material was coarse than that sampled under reference conditions; nevertheless, largest particles settled quickly, suggesting a selective transport downstream. This study constitutes a first step towards a better understanding of local sediment dynamics in rivers affected by maintenance works and related activities, such as gravel mining, and, overall, supports the comprehensive assessment of the effects of human actions on channel morphodynamics and the ecological functioning of mountain fluvial systems.
\end{abstract}

Palabras clave: actuaciones en cauces, extracción de áridos, sedimento en suspensión, granulometría, sedimentación, río Cinca.

Key words: channel maintenance works, gravel mining, suspended sediment transport, grain-size distribution, deposition, River Cinca.

Recibido: 10 de febrero de 2017

Aceptado: 4 de julio de 2017

* Correspondencia: M. Béjar, Fluvial Dynamics Research Group (RIUS), Universitat de Lleida, Lleida, España. E-mail: mariabejar@macs.udl.cat

\section{Introducción}

Los ríos mantienen un balance sedimentario donde la cantidad de sedimento procedente de las zonas de erosión es equivalente a la cantidad de sedimento que reciben las zonas de sedimentación. Por tanto, la transferencia de sedimentos es un proceso continuo a lo largo de la cuenca y es el resultado de las características hidroclimáticas, litológicas, edafológicas y vegetales, así como de los procesos 
biofísicos que tienen lugar en la misma (generación de sedimentos, erosión y transporte). Sin embargo, las actividades humanas (i.e. presas y actuaciones en el cauce como la construcción de escolleras y las extracciones de áridos) y otros procesos, como los cambios en los usos del suelo de la cuenca, alteran esta transferencia de sedimentos aguas abajo (Williams y Wolman, 1984; García-Anquela et al., 1985; Kondolf, 1994).

Las arenas y gravas transportadas por los ríos se utilizan para la construcción (i.e. áridos) y se extraen en muchos casos de los depósitos aluviales (terrazas, llanura de inundación) o directamente del cauce fluvial. Como consecuencia, las extracciones de áridos alteran la transferencia de sedimento aguas abajo generando un déficit de las fracciones de mayor tamaño (aquellas que forman la arquitectura básica del cauce) e incrementando proporcionalmente la disponibilidad de sedimento fino. Los principales efectos descritos en la literatura incluyen: la alteración de la geometría del canal, la disminución del nivel freático debido al sobredrenaje, el incremento del tamaño de partículas del lecho y el incremento de sedimento fino (p.ej., Kondolf, 1994). Se han realizado numerosos estudios de los efectos geomorfológicos de las extracciones de áridos (Rinaldi et al., 2005; Rovira et al., 2005), pero son escasos los trabajos que han analizado específicamente el efecto de estas actuaciones en el transporte de sedimentos finos en suspensión (arcilla, limo e incluso arena; Brown et al., 1998). Durante las operaciones de extracción aumenta el material en suspensión debido a la erosión lateral al extraer material del canal (Warner et al., 1977), al paso de maquinaria por el cauce del río (Forshage y Carter, 1973) y a la remoción de materiales y exposición de sedimentos finos al romper la coraza del lecho (Lagasse et al., 1980). Los efectos no son localizados y se pueden extender en el espacio y en el tiempo (kilómetros aguas abajo e incluso aguas arriba, durante años y décadas) hasta que el río alcanza una nueva condición de equilibrio (Kondolf, 1997).

El incremento de la carga de sedimento fino comporta una notable perturbación de los ecosistemas acuáticos (Wood y Armitage, 1997). Este incremento puede afectar directamente a los organismos e indirectamente al hábitat físico (Jones et al., 2012). Las partículas en suspensión pueden colapsar las agallas de los organismos afectando a la eficiencia de respiración (Bilotta y Brazier, 2008). También pueden causar abrasión sobre los animales (Newcombe y Macdonald, 1991). Además, el sedimento en suspensión se sedimenta aguas abajo alterando las características del sustrato y por tanto la capacidad de refugio de los animales (Buendía et al., 2011), así como procesos esenciales para el ecosistema como la freza de salmónidos (Waters, 1995). La presencia de sedimento también puede alterar los niveles de oxígeno y desencadenar otros cambios en la composición química del agua (Pretty et al., 2006). Del mismo modo, el aumento de la turbidez puede desencadenar cambios físicos como la reducción de la penetración de luz, limitando el crecimiento algal (Parkhill y Gulliver, 2002). Por tanto, cuantificar el impacto de estas actuaciones en la dinámica hidro-sedimentaria es fundamental para entender la dinámica en los ríos afectados por actividades humanas y, en la medida de lo posible, aportar información a los organismos gestores para que puedan anticiparse a los posibles efectos ambientales y diseñar e implementar las actuaciones de mitigación necesarias dentro de los 
programas de gestión de cuencas. En este contexto, el objetivo de este trabajo es analizar los efectos de una extracción de áridos sobre el transporte de sedimento en suspensión, evaluando los cambios de las concentraciones, las cargas sedimentarias y las características granulométricas durante la actuación, y determinando el alcance espacial de dicho impacto. El estudio se realiza en un tramo del alto Cinca (Pirineo Central), afectado históricamente y hasta la actualidad por distintos tipos de actuaciones en el cauce, singularmente extracciones de áridos. Cabe destacar que la extracción de áridos monitorizada en este estudio se realiza en el marco de una actuación en el cauce para la prevención de inundaciones.

\section{2. Área de estudio}

\subsection{El tramo alto del Cinca}

El río Cinca tiene un régimen nivo-pluvial y es uno de los mayores tributarios del Ebro. El tramo alto del río Cinca se sitúa en el noreste de la Península Ibérica en la vertiente sur de los Pirineos (Fig. 1A). La cuenca del alto Cinca tiene una superficie de $848 \mathrm{~km}^{2}$ y un gradiente altitudinal que se extiende desde Monte Perdido (3355 m s.n.m.) hasta Ainsa (522 m s.n.m.; Fig. 1B). La aportación media anual de agua es de $587 \mathrm{hm}^{3}$, lo que supone un caudal medio en el tramo de estudio de $29 \mathrm{~m}^{3} / \mathrm{s}$ (según datos en la estación de aforos A051 de Escalona entre 1959-2015; ver Fig. 1C). El tramo está sujeto a hidropicos esporádicos que no exceden los $25 \mathrm{~m}^{3} / \mathrm{s}$ (i.e. originados desde la central hidroeléctrica de Laspuña, $8 \mathrm{~km}$ aguas arriba del tramo de estudio). Estos hidropicos alteran la hidráulica del canal pero no son suficientemente competentes para arrastrar materiales del lecho. La magnitud de las crecidas anuales excede $\operatorname{los} 225 \mathrm{~m}^{3} / \mathrm{s}$. La pendiente media del tramo de estudio es $0,007 \mathrm{~m} / \mathrm{m}$, el ancho del canal activo promedia $200 \mathrm{~m}$ y la profundidad media es $0,4 \mathrm{~m}$ para caudales bajos (i.e. $10 \mathrm{~m}^{3} / \mathrm{s}$ ). En las últimas décadas la morfología del tramo ha experimentado una reducción en su complejidad, pasando de un patrón trenzado a un patrón sinuoso, con canales más estables (Llena et al., 2016). Los sedimentos del lecho están poco clasificados (i.e. Índice de dispersión $\mathrm{I}_{\mathrm{F} \& W}=1,1$; Folk y Ward 1957), el rango de partículas alcanza desde grandes bloques a gravas finas (> 2000 a $<8 \mathrm{~mm}$ ). La mediana de la distribución granulométrica superficial $\left(\mathrm{D}_{50-\mathrm{s}}\right)$ es de $67 \mathrm{~mm}$ (i.e. río de transición entre gravas y cantos). La concentración media de sedimentos en suspensión para el periodo 2013-2015 es 0,09 g/l, alcanzando un máximo instantáneo de $17 \mathrm{~g} / 1$ asociado a un caudal de $48 \mathrm{~m}^{3} / \mathrm{s}$ durante la crecida del 31/07/2015. Se trata de una concentración similar a las obtenidas por Lobera et al. (2016a, 2016b) en la vecina cuenca del río Ésera. 


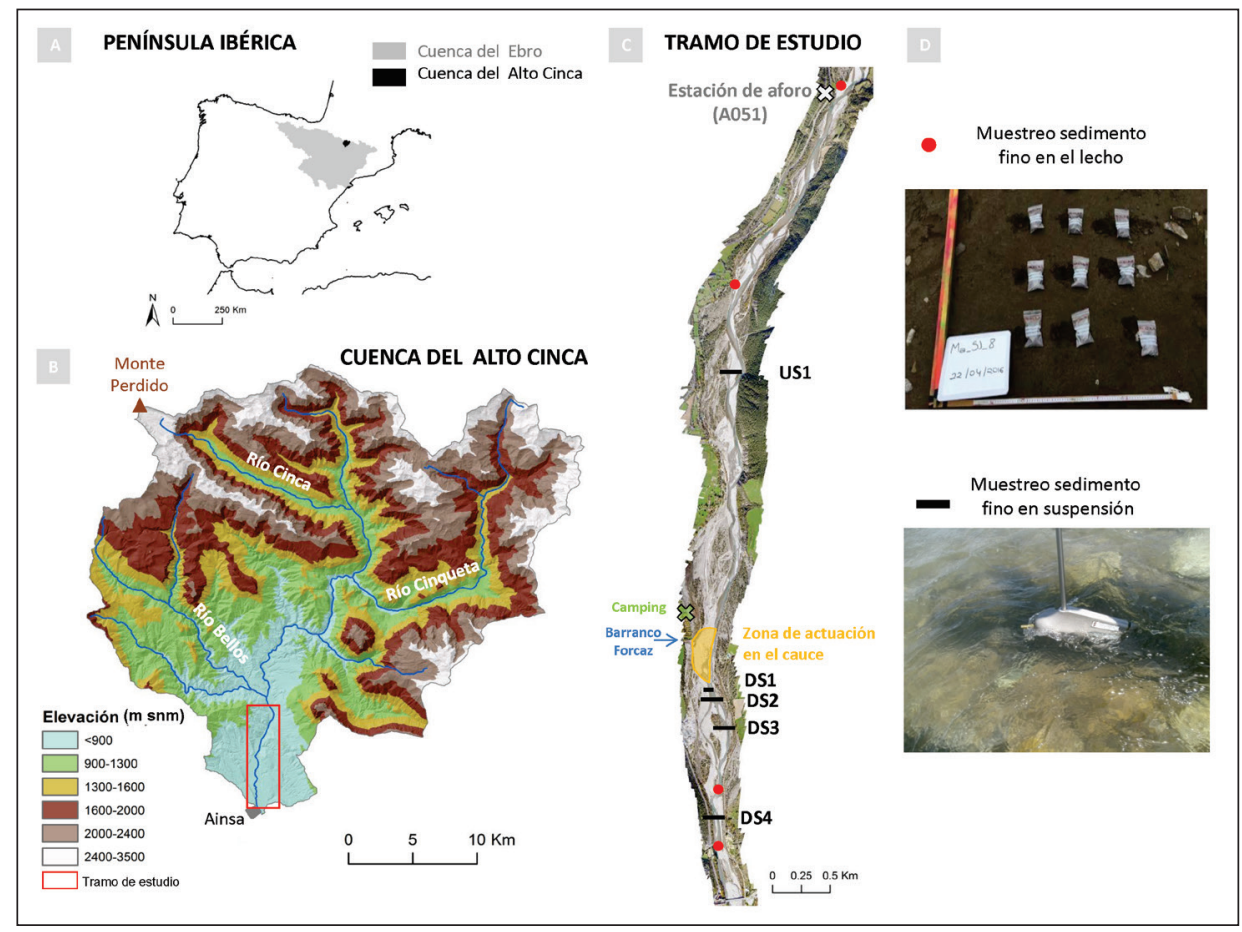

Figura 1. Área de estudio y métodos aplicados. (A) Localización de la cuenca del alto Cinca en la Península Ibérica. (B) Localización del tramo de estudio (rectángulo rojo) en la cuenca del alto Cinca. (C) Tramo de estudio y localización de las secciones y puntos de muestreo. (D) Detalle del muestreo de sedimento fino en el lecho y la adquisición de sedimento en suspensión mediante un tomamuestras de integración en profundidad US DH48.

\subsection{Extracciones de áridos}

El alto Cinca ha experimentado extracciones de áridos desde mediados del siglo XX. En la actualidad las extracciones de áridos se enmarcan dentro de las actuaciones en el cauce activo para la prevención y mitigación de los efectos de las crecidas (i.e. defensas de márgenes, re-ubicación del cauce). La actuación estudiada tuvo lugar en agosto de 2014 en el término municipal de Labuerda. La zona de la actuación se localiza en la desembocadura de un afluente, barranco Forcas, donde, además, el cauce se encuentra próximo a un camping (Fig. 2A). La figura 2 ilustra las distintas fases de la actuación en base a los objetivos de la misma: (a) alejar el cauce activo del camping y (b) extraer los sedimentos ubicados en una barra activa del cauce para disminuir la cota del cauce y reducir así el riesgo de inundación. La actuación se programó en tres fases: 1) una primera fase de preparación de un cauce alternativo (Fig. 2B), en la que se extrajeron sedimentos para la creación del cauce alternativo; 2) una segunda fase donde se conectó el extremo superior del cauce alternativo con el cauce principal y se derivó el agua hacia el nuevo cauce (Fig. 2C); y, finalmente, 3) una tercera fase en la que se extrajo material 
de la barra activa del cauce (Fig. 2D). El aporte de sedimento fino se produjo sobre todo durante el primer día de la primera fase (día 6; Fig. 3A) y durante el primer día de la segunda fase (día 18; Fig. 3B). La tercera fase se llevó a cabo en cauce seco y por tanto no aportó sedimento fino de manera directa al cauce.

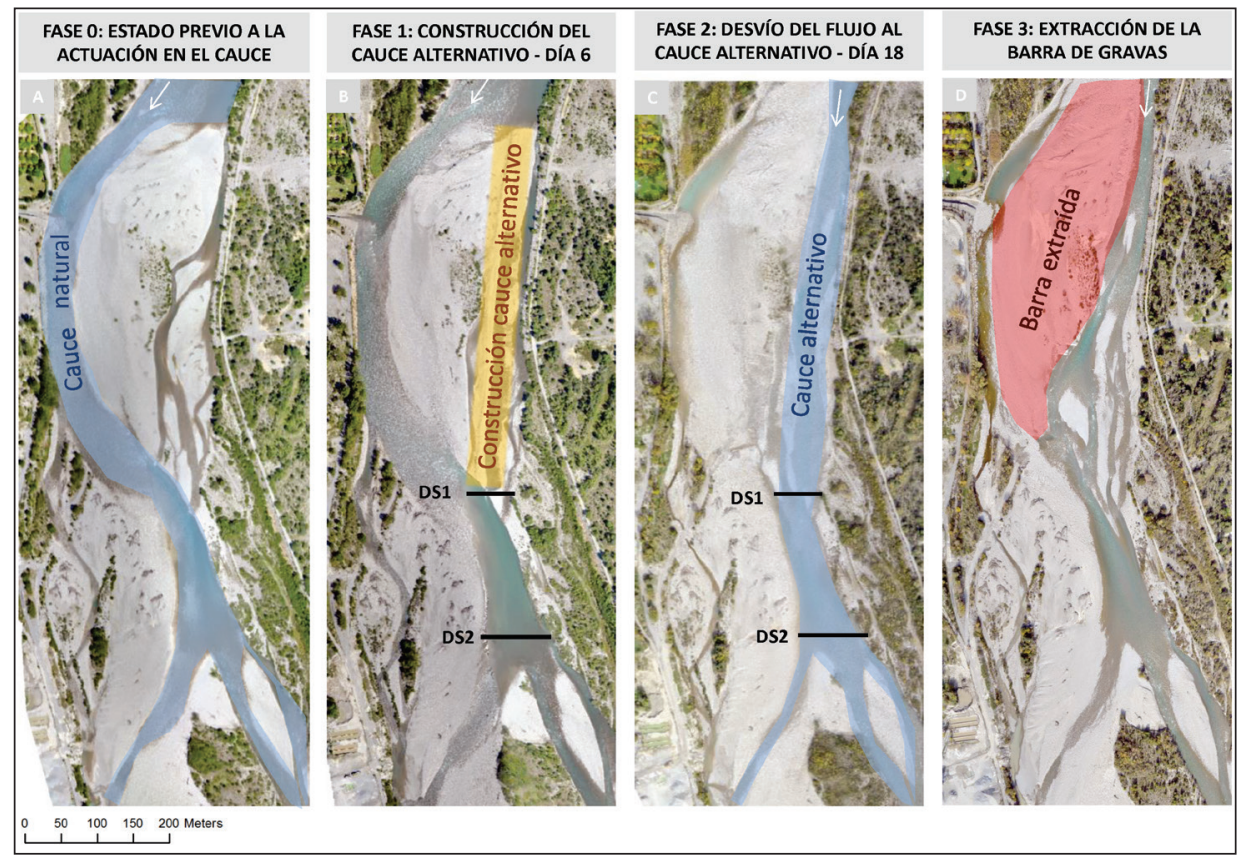

Figura 2. Fases de la actuación analizada en un tramo del alto Cinca en agosto de 2014. (A) Fase 0: Estado previo de la actuación. (B) Fase 1: Construcción del cauce alternativo en el margen izquierdo. Esta fase implica la extracción de materiales para dicha construcción. $(C)$ Fase 2: Apertura del cauce alternativo y desviación del flujo. (D) Fase 3: Extracción de áridos en la barra diagonal. Nota: La flecha blanca indica la dirección del flujo.

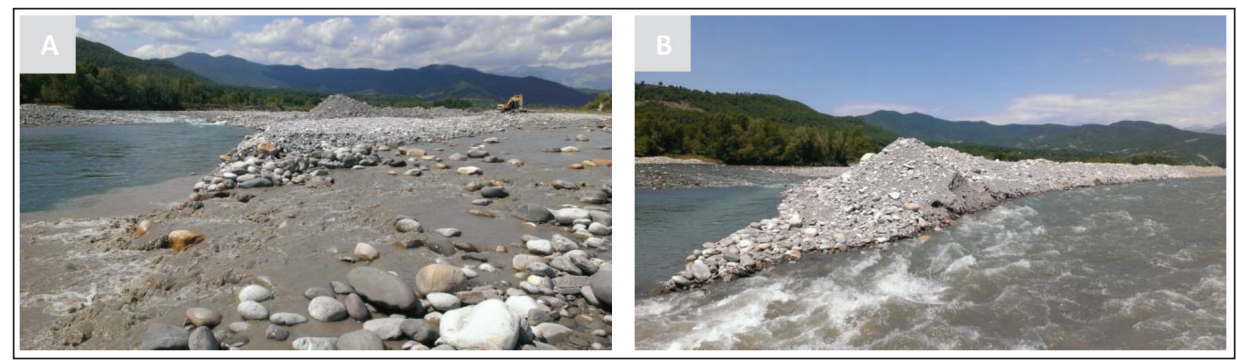

Figura 3. Fotografías durante los trabajos de adecuación previos a la actuación en el cauce (extracción de áridos) en un tramo del alto Cinca en agosto de 2014. (A) Pluma de sedimento en suspensión durante la creación del cauce alternativo el día 6 de agosto, corresponde a la máxima concentración registrada. (B) Detalle de la erosión lateral durante la apertura del cauce alternativo el día 18 de agosto. 


\section{Métodos}

\subsection{Caudal e hidráulica}

El caudal se obtuvo a partir de los registros de la estación de aforos de Escalona (A051, Confederación Hidrográfica del Ebro) ubicada 1,5 km aguas arriba de la zona de actuación (Fig. 1C). Un limnígrafo obtiene medidas cada quince minutos de nivel de agua (h) que posteriormente se transforman a caudal $(\mathrm{Q})$ mediante una curva de gasto h/Q. El modelo hidráulico 2D Iber ${ }^{\circledR}$ ha sido utilizado en el tramo de estudio para caracterizar la hidráulica durante la extracción. El modelo utiliza datos topográficos y ecuaciones de dinámica de fluidos para simular la lámina de agua en ríos y estuarios (para más detalles consultar Bladé et al., 2014). La parametrización del modelo se ha realizado usando un Modelo Digital del Terreno de $1 \mathrm{~m}$ de resolución (ver Vericat et al., 2016) y un solo valor de rugosidad $(n=0,035)$ basado en los datos granulométricos del tramo. La modelización se ha hecho para un caudal constante $7 \mathrm{~m}^{3} / \mathrm{s}$ con el objetivo de obtener valores de hidráulica característicos en el tramo.

\subsection{Medición del transporte de sedimentos}

El muestreo del sedimento en suspensión se llevó a cabo en cinco secciones del tramo de estudio, una sección aguas arriba (US1) y cuatro aguas abajo (DS1-4; Fig. 1C). La sección aguas arriba se utilizó como control. La primera sección aguas abajo (DS1) se localizó inmediatamente aguas abajo de la zona alterada. Las secciones restantes se situaron a 0,2, 0,5 y 1,5 km de la zona de actuación. Durante los dos días en los que el cauce fue alterado (día 6 y día 18), se realizó un muestreo horario en las secciones más próximas a la extracción (DS1-DS3). El muestreo se llevó a cabo mediante un muestreador de integración en profundidad US DH48 (Fig. 1D) en aquellas secciones donde las diferencias de turbidez en la sección eran evidentes (DS1-DS3). Cada muestra se compuso de tres sub-muestras tomadas en diferentes puntos a lo ancho de la sección. De este modo, las muestras representan las concentraciones medias en la sección ya que cada muestra integra la $\mathrm{C}_{\mathrm{SS}}$ en tres puntos representativos a lo ancho de la sección y cada punto integra la $\mathrm{C}_{\mathrm{SS}}$ en la columna de agua (i.e. vertical). En las secciones US1 y DS4, donde el transporte era más homogéneo, se muestreó únicamente en el centro de la sección con un intervalo de tiempo mayor entre muestras (3-4 horas). En total se obtuvieron 300 muestras de 1 1. Las muestras se filtraron (filtros Filter-Lab, 1,2 $\mu \mathrm{m}$ tamaño de poro), se secaron y se pesaron en el laboratorio para determinar la concentración de sedimentos en suspensión $\left(\mathrm{C}_{\mathrm{SS}}\right.$ en $\left.\mathrm{g} / \mathrm{l}\right)$.

\subsection{Obtención de las granulometrías de los sedimentos finos}

Las muestras retenidas en los filtros fueron re-suspendidas en agua para su posterior análisis por vía húmeda. La granulometría se determinó usando un analizador láser de tamaño de partículas (Malvern Mastersizer $\left.{ }^{\circledR} / \mathrm{E}\right)$, que permite analizar la distribución granulométrica de las fracciones entre $0,02 \mu \mathrm{m}$ (arcillas) y $500 \mu \mathrm{m}$ (arenas medias). Del total de las muestras obtenidas se caracterizaron setenta y dos (localizadas en DS1-DS3). El resto no se pudieron analizar debido a la falta de material suficiente para su detección. 
Además, se tomaron muestras granulométricas del lecho en cuatro puntos a lo largo del tramo de estudio y en una sola ocasión (22 de abril de 2016). En total, se muestrearon dos puntos aguas arriba y dos puntos aguas abajo de la zona de actuación (Fig. 1C). En cada punto se seleccionó un parche superficial de sedimento fino y se tomaron 10 réplicas del material (Fig. 1D). En total se tomaron cuarenta muestras de aproximadamente $15 \mathrm{~g}$ cada una. Las muestras se secaron, tamizaron y pesaron. Las partículas de $2000 \mu \mathrm{m}$ (arenas muy gruesas) a $500 \mu \mathrm{m}$ (arenas medias) se tamizaron en una torre granulométrica de la marca Filtra®. Las partículas más finas de $500 \mu \mathrm{m}$ se dispersaron en agua agitando durante seis horas y posteriormente se procesaron siguiendo el mismo procedimiento detallado anteriormente.

\subsection{Análisis de datos}

El cálculo de la carga sólida total $\left(\mathrm{C}_{\mathrm{ST}}\right)$ se realizó para el intervalo de 09:00 a 19:00 horas ya que son las horas de trabajo en la zona de extracción. Para cada sección de muestreo, la carga total se calculó como la suma de las cargas de cada intervalo de muestra de agua $\left(\mathrm{C}_{\mathrm{ST} t=\mathrm{i}}\right)$. Para ello $\mathrm{C}_{\mathrm{ST} t=1}(\mathrm{t})$ fue calculado multiplicando la $\mathrm{C}_{\mathrm{SS} t=1}(\mathrm{~g} / \mathrm{l})$ obtenida de cada muestra de integración en profundidad por el caudal circulante $\left(\mathrm{Q}_{\mathrm{t}=1}\right.$; $1 / \mathrm{s})$ por el intervalo de tiempo entre muestras $\left(\mathrm{t}_{2}-\mathrm{t}_{1}, \mathrm{~s}\right)$.

Durante el día 6 y en el caso particular de DS1, se tuvieron que realizar cálculos proporcionales de caudal ya que la sección estaba inicialmente dividida en un brazo principal (el canal natural) y uno secundario (donde se construiría el cauce alternativo; ver Fig. 2B). De este modo, durante el día 6 se estimó un caudal circulante en el brazo secundario (basado en observaciones de campo) del $10 \%$ del caudal total. En esta misma sección, el día 18 el caudal varió a medida que se desviaba el flujo hacia el cauce alternativo (Fig. 2C). Por tanto, los cálculos se han realizado en base al caudal aproximado que se estimó en campo (observaciones de anchura, profundidad y velocidad superficial) para cada intervalo de muestreo. El caudal representaba el $10 \%$ del total al inicio del muestreo, mientras que al finalizar el muestreo el caudal en el cauce alternativo representaba el 90\% del total.

Las distribuciones granulométricas de las muestras de sedimento fino del lecho se truncaron por ambos extremos elaborándolas para el intervalo 1,2-2000 $\mu \mathrm{m}$. De este modo los valores estadísticos de las curvas son comparables con las distribuciones granulométricas del material en suspensión muestreado durante las actuaciones en el cauce.

\section{Resultados y discusión}

\subsection{Hidrología e hidráulica}

Durante el mes de los trabajos de adecuación y extracción (agosto de 2014), el caudal medio fue relativamente bajo y estable (igualado o excedido el $85 \%$ del tiempo en la curva de frecuencia de caudal para el período 1959-2016, datos sin publicar), cercano a los $9 \mathrm{~m}^{3} / \mathrm{s}(\mathrm{CV}=0,4$, donde $\mathrm{CV}$ es el coeficiente de variación). El caudal para todo el año hidrológico 2013-2014 osciló entre $5,2 \mathrm{~m}^{3} / \mathrm{s}$ y $477 \mathrm{~m}^{3} / \mathrm{s}$ (representado éste último valor un caudal pico de crecida con un periodo de retorno de 5 años según Llena et al., 2016). Los caudales durante los días de estudio (días 6 y 18) fueron 6,9 y 7,0 m³ 
respectivamente (para más detalles ver Béjar et al. 2017). Estos valores son igualados o superados un 93\% del tiempo. Los resultados preliminares (datos sin publicar) del modelo hidráulico 2D Iber ${ }^{\circledR}$ indican que la profundidad media del cauce en todo el tramo de estudio durante los días 6 y 18 fue de $0,3 \mathrm{~m}(\mathrm{CV}=0,7 \mathrm{~m})$, y la velocidad media $0,7 \mathrm{~m} / \mathrm{s}(\mathrm{CV}=0,6)$. Los caudales de esos días no produjeron ningún movimiento apreciable del material grueso del lecho.

\subsection{Transporte de sedimentos}

\subsubsection{Dinámica temporal y espacial}

La $\mathrm{C}_{\mathrm{ss}}$ en la sección de control (i.e. US1) osciló entre 2 y $50 \mathrm{mg} / 1$, con un promedio de $20 \mathrm{mg} / \mathrm{l}(\mathrm{CV}=1,2)$ durante los días 6 y 18 (Tabla 1). Estos valores se consideran normales si se comparan con los promedios obtenidos en DS4 para los seis meses anteriores a la actuación (41 mg/l, datos sin publicar). En cambio, aguas abajo de la actuación, la $\mathrm{C}_{\mathrm{ss}}$ media fue un orden de magnitud mayor y mostró una mayor variabilidad $\left(\mathrm{C}_{\mathrm{ss}}=0,2 \mathrm{~g} / \mathrm{l} ; \mathrm{CV}=3,8\right)$.

Durante el día 6 la $\mathrm{C}_{\mathrm{ss}}$ incrementa puntualmente en la sección más próxima a la actuación (DS1), alcanzándose un máximo de 5,8 g/l (Fig. 4A; Tabla 1). Este incremento se debe probablemente a la liberación de materiales finos durante la construcción del cauce alternativo en el margen izquierdo (Fig. 3A). En las siguientes secciones (DS2, DS3, DS4), las concentraciones fueron menores alcanzándose máximos de 0,07, 0,02, y 0,02 g/l respectivamente (Tabla 1). La disminución de casi dos órdenes de magnitud en las $\mathrm{C}_{\mathrm{SS}}$ entre DS1 y DS2 (a 200 m de distancia) indica una probable sedimentación de la mayoría del material movilizado durante las actuaciones llevadas a cabo en el cauce en ese día.

Tabla 1. Concentración de sedimento en suspensión $\left(C_{S S}\right)$ analizado durante una extracción en el alto Cinca en agosto de 2014.

\begin{tabular}{|c|cccc|}
\hline \multirow{2}{*}{ Día } & \multirow{3}{*}{ Sección $^{1}$} & \multicolumn{3}{c|}{ Concentración de Sedimentos en Suspensión (g/l) } \\
\cline { 2 - 5 } & & $\mathbf{C}_{\mathrm{SS}}$ media & $\mathbf{C}_{\mathrm{SS}}$ máxima & $\mathbf{C}_{\mathrm{SS}}$ mínima $^{*}$ \\
\hline \multirow{3}{*}{$06 / 08 / 2014$} & US1 & $0,004^{2}$ & 0,004 & 0,004 \\
& DS1 & 0,419 & 5,806 & 0,014 \\
& DS2 & 0,017 & 0,070 & 0,006 \\
& DS3 & 0,009 & 0,022 & 0,006 \\
& DS4 & 0,008 & 0,023 & 0,004 \\
\hline \multirow{3}{*}{$18 / 08 / 2014$} & US1 & 0,021 & 0,054 & 0,010 \\
& DS1 & 0,181 & 0,610 & 0,002 \\
& DS2 & 0,122 & 0,842 & 0,002 \\
& DS3 & 0,158 & 1,341 & 0,005 \\
& DS4 & 0,002 & 0,003 & 0,001 \\
\hline
\end{tabular}

\footnotetext{
${ }^{1}$ Ver la figura 1 para localización de las secciones.

${ }^{2}$ Solo se tomó una muestra, por tanto solo se tiene un valor de $\mathrm{C}_{\mathrm{SS}}$
} 


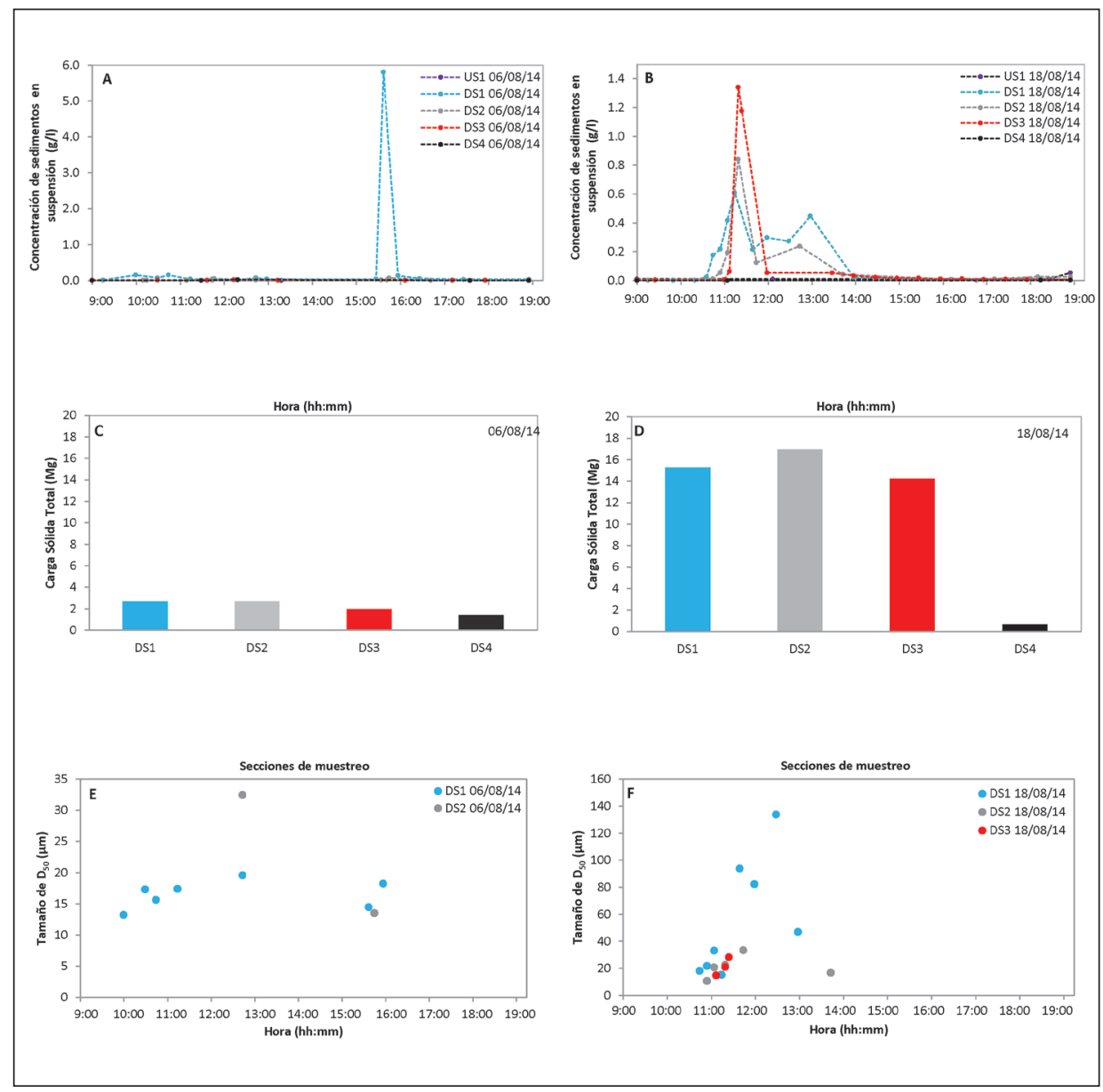

Figura 4. Transporte de sedimento en suspensión durante los trabajos asociados a la extracción de áridos en las secciones de muestreo localizadas aguas abajo de la extracción. (A) Concentración de sólidos en suspensión durante el día 6 de agosto. (B) Concentración de sólidos en suspensión durante el día 18 de agosto (Nota: el eje vertical está modificado). (C) Carga sólida en suspensión durante el día 6 de agosto. (D) Carga sólida en suspensión durante el día 18 de agosto. (E) Tamaño medio de las partículas en suspensión durante el día 6 de agosto. (F) Tamaño medio de las partículas en suspensión durante el día 18 de agosto.

Durante el día 18 se observó una dinámica completamente diferente (Fig. 4B). La $\mathrm{C}_{\mathrm{ss}}$ incrementó aguas abajo registrándose el máximo $(1,30 \mathrm{~g} / \mathrm{l})$ en la sección situada a $0,5 \mathrm{~km}$ de la actuación (DS3). Nuestra hipótesis es que esta recarga aguas abajo durante el día 18 responde a la movilización y tránsito de los materiales en el lecho producto de la fuerte sedimentación observada el día 6 , y en gran medida relacionada con la apertura del cauce alternativo que moviliza el sedimento disponible en el lecho debido a la concentración del flujo (Fig. 2C y 3B). En la sección más alejada (DS4), 
la $\mathrm{C}_{\mathrm{SS}}$ máxima fue $0,003 \mathrm{~g} / \mathrm{l}$. El patrón en DS4 es similar al observado en el día 6, indicando que el sedimento movilizado no se mantiene en suspensión a lo largo de los $1,5 \mathrm{~km}$ del tramo de estudio. Otros autores han reportado el mismo efecto, con plumas de sedimento durante actuaciones en el cauce en condiciones de caudal estable a lo largo de los primeros $500 \mathrm{~m}$ (p.ej. Bryce, 1977; Mori y Braceli, 2011).

Las $\mathrm{C}_{\mathrm{SS}}$ medias alcanzadas durante los días de estudio son similares a los valores registrados durante crecidas naturales (p.ej. la $\mathrm{C}_{\mathrm{SS}}$ media durante un evento el 02/08/14 fue de $0,4 \mathrm{~g} / \mathrm{l})$. Sin embargo, la $\mathrm{C}_{\mathrm{sS}}$ máxima durante el periodo de estudio fue el doble que la $\mathrm{C}_{\mathrm{sS}}$ máxima alcanzada en el mismo evento (i.e. 2,6 g/l). Si se comparan la $\mathrm{C}_{\mathrm{ss}}$ máxima obtenidas durante las actuaciones en el cauce con las obtenidas en eventos de crecida en el cercano río Ésera, la $\mathrm{C}_{\mathrm{SS}}$ máxima es mucho menor (i.e. $\mathrm{C}_{\mathrm{SS}}$ máxima en verano 27,8 g/l, Lobera et al., 2016b). Es interesante señalar también que las altas concentraciones alcanzadas durante los días de actuación en el cauce alteraron por ejemplo la deriva de macroinvertebrados en el tramo de estudio (para más detalles consultar Béjar et al., 2017).

\subsubsection{Carga sólida}

La carga sólida $\left(\mathrm{C}_{\mathrm{ST}}\right)$ diaria durante los dos días de muestreo varió entre 0,7 y $17 \mathrm{Mg}$. En la sección de referencia (US1) se registró una media de 1,8 $\mathrm{Mg}(\mathrm{CV}=0,7)$, un orden de magnitud inferior que el valor máximo observado aguas abajo de la actuación.

Durante el día 6 la $\mathrm{C}_{\mathrm{ST}}$ media fue 2,2 $\mathrm{Mg}$ en las secciones situadas aguas abajo de la extracción (Fig. 4C). El máximo (2,7 Mg) se registró en los primeros 0,2 km (DS1 y DS2). Sin embargo, es importante remarcar que durante este día el caudal circulante por DS1 representaba sólo un 10\% del caudal total (Fig. 3A), hecho que demuestra el diferente grado de impacto de las actuaciones en el cauce en la carga sedimentaria en ambas secciones. Durante el día 18 la carga total media en las secciones DS1 a DS4 fue un orden de magnitud superior al estimado durante el día 6 (11,8 Mg, Fig. 4D). La carga sólida circulante por las secciones situadas en los primeros 0,5 km (DS1-DS3) fue similar $(\mathrm{CV}=0,1)$ y el máximo de carga se alcanzó en DS2 $(17 \mathrm{Mg})$.

Es interesante señalar que mientras la $\mathrm{C}_{\mathrm{SS}}$ máxima se registró en DS1 el día 6 (sección 4.2.1) la $\mathrm{C}_{\mathrm{ST}}$ máxima se observó en DS2 durante el día 18. En la figura 4A se muestra como la $\mathrm{C}_{\mathrm{SS}}$ máxima el día 6 es un valor puntual precedido y sucedido por valores próximos a 0 . Sin embargo, en la figura $4 \mathrm{~B}$ se observa como las $\mathrm{C}_{\mathrm{SS}}$ durante el día 18 fueron de menor magnitud, pero con una concentración media elevada y mantenida en el tiempo (hasta 4 horas). Este hecho tiene efectos en el balance sedimentario pero también en la vida acuática. Newcombe y Macdonald (1991) ya demostraron cómo la biota acuática responde tanto a los cambios puntuales en la $\mathrm{C}_{\mathrm{SS}}$ como a la duración de la exposición. En el caso del Cinca dichos efectos se describen en el trabajo recientemente publicado por Béjar et al. (2017).

La carga media durante los dos días de actuación en la sección más lejana de la actuación (DS4) fue de $1 \mathrm{Mg}$, valor similar al obtenido en la sección de referencia (US1) para el mismo periodo. Este hecho sugiere que la pluma de sedimento generada 
por las maquinas al extraer gravas no impactó a DS4, indicando que todo el material movilizado durante los días 6 y 18 se sedimentó en el cauce intermedio entre secciones, quedando como una fuente local de sedimento para próximas crecidas. La carga máxima registrada durante los días de estudio representa un $10 \%$ de la carga alcanzada durante eventos naturales en la misma época del año. Por ejemplo, la carga movilizada durante el evento del 02/08/14 en condiciones similares de duración y caudal (10 horas, caudal medio $12 \mathrm{~m}^{3} / \mathrm{s}$ ) fue de $227 \mathrm{Mg}$.

\subsection{Textura del sedimento en suspensión}

El sedimento movilizado durante el día 6 en las secciones más próximas a la actuación presentó una granulometría limosa $\left(\mathrm{D}_{50 \text {-medio }}=18 \mu \mathrm{m}\right.$, con un rango entre 13 y $33 \mu \mathrm{m}$; Tabla 2). El $\mathrm{D}_{50}$ máximo se obtuvo en la sección DS2 durante un periodo de baja concentración (Fig. 4E). Durante el día 18, la distribución del tamaño de partículas fue más variable en el tiempo y también entre secciones (Fig. 4F). En la sección más próxima a la actuación (DS1) fue donde se observó una mayor heterogeneidad granulométrica $\left(\mathrm{I}_{\mathrm{F} \& \mathrm{~W}}=2,6\right.$; Tabla 1$)$ a partir de la apertura del cauce alternativo, así como un incremento en el tamaño medio de partícula, pasando de limos a arenas finas a medida que transcurría la intervención en el cauce (Tabla 2). Estos resultados responden a los procesos de erosión y transporte observados tras la apertura del cauce alternativo (Fig. 3B), que movilizaron materiales peor clasificados y más gruesos que los que normalmente se mueven en suspensión durante crecidas naturales. En las secciones más alejadas (DS2 y DS3) el rango del tamaño medio de partículas es similar al observado durante el día 6 (11-34 $\mu$ m; Tabla 2). Además, en estas dos secciones se observa una tendencia a la homogeneización granulométrica con tan solo un valor de $I_{F \& W}$ por encima del límite entre clasificación pobre y muy pobre $\left(I_{\mathrm{F} \& \mathrm{~W}}=2,1\right)$. Globalmente los resultados indican un lavado selectivo de los materiales transportados aguas abajo (i.e. las partículas de mayor tamaño tienden a sedimentar en los primeros metros aguas abajo del impacto como consecuencia de la falta de caudales competentes para su transporte). Es importante destacar este efecto en la dinámica espacial de las concentraciones durante los dos pulsos del día 18 (Fig. 4B): el primer pulso, con partículas más finas (Fig. 4F), alcanza las secciones más alejadas y además se produce una movilización del sedimento depositado en los días previos, lo que condicionaría la magnitud de las $\mathrm{C}_{\mathrm{ss}}$; $\sin$ embargo, durante el segundo pulso, las partículas tienen un mayor tamaño y el incremento de $\mathrm{C}_{\mathrm{SS}}$ no alcanzó las secciones más alejadas debido al lavado selectivo (Fig. 4F). 
Tabla 2. Descriptores sedimentológicos del material en suspensión analizado durante una extracción en el alto Cinca en agosto de 2014.

\begin{tabular}{|c|c|c|c|c|c|c|c|}
\hline Día & Sección & Hora & $\begin{array}{c}\mathrm{D}_{50} \\
(\mu \mathrm{m})\end{array}$ & $\begin{array}{c}\text { Velocidad } \\
\text { Sedimentación }^{1} \\
(\mathrm{~cm} / \mathrm{s})\end{array}$ & $\begin{array}{l}\text { Clasificación } \\
\text { Wentworth }\end{array}$ & $\begin{array}{c}\text { Índice de } \\
\text { dispersión }^{2}\end{array}$ & $\begin{array}{c}\text { Grado de } \\
\text { clasificación }\end{array}$ \\
\hline \multirow{9}{*}{$06 / 08 / 14$} & \multirow{7}{*}{ DS1 } & $10: 00$ & 13,2 & 0,06 & Limo & 1,43 & Pobre \\
\hline & & $10: 30$ & 17,3 & 0,09 & Limo & 1,50 & Pobre \\
\hline & & $10: 45$ & 15,6 & 0,08 & Limo & 1,53 & Pobre \\
\hline & & $11: 15$ & 17,4 & 0,10 & Limo & 1,43 & Pobre \\
\hline & & $12: 45$ & 19,6 & 0,12 & Limo & 1,63 & Pobre \\
\hline & & $15: 40$ & 14,5 & 0,07 & Limo & 1,49 & Pobre \\
\hline & & $16: 00$ & 18,2 & 0,10 & Limo & 1,75 & Pobre \\
\hline & \multirow{2}{*}{ DS2 } & $12: 45$ & 32,5 & 0,33 & Limo & 1,81 & Pobre \\
\hline & & $15: 48$ & 13,5 & 0,06 & Limo & 1,44 & Pobre \\
\hline \multirow{16}{*}{$18 / 08 / 14$} & \multirow{8}{*}{ DS1 } & $10: 45$ & 18,1 & 0,10 & Limo & 1,56 & Pobre \\
\hline & & $10: 55$ & 21,8 & 0,15 & Limo & 1,75 & Pobre \\
\hline & & $11: 05$ & 33,1 & 0,35 & Limo & 2,05 & Muy pobre \\
\hline & & $11: 15$ & 15,2 & 0,07 & Limo & 1,99 & Muy pobre \\
\hline & & $11: 40$ & 93,7 & 2,77 & Arenas muy finas & 2,53 & Muy pobre \\
\hline & & $12: 00$ & 82,2 & 2,13 & Arenas muy finas & 2,61 & Muy pobre \\
\hline & & $12: 30$ & 133,8 & 5,65 & Arenas finas & 2,63 & Muy pobre \\
\hline & & $13: 00$ & 46,9 & 0,69 & Limo & 2,63 & Muy pobre \\
\hline & \multirow{5}{*}{ DS2 } & $10: 55$ & 10,7 & 0,04 & Limo & 1,39 & Pobre \\
\hline & & $11: 05$ & 20,7 & 0,13 & Limo & 1,48 & Pobre \\
\hline & & $11: 20$ & 22,6 & 0,16 & Limo & 1,70 & Pobre \\
\hline & & $11: 45$ & 33,5 & 0,35 & Limo & 2,05 & Muy pobre \\
\hline & & $13: 45$ & 16,7 & 0,09 & Limo & 1,85 & Pobre \\
\hline & \multirow{3}{*}{ DS3 } & $11: 08$ & 14,9 & 0,07 & Limo & 1,85 & Pobre \\
\hline & & $11: 20$ & 21,0 & 0,14 & Limo & 1,57 & Pobre \\
\hline & & $11: 25$ & 28,3 & 0,25 & Limo & 1,51 & Pobre \\
\hline
\end{tabular}

${ }^{1}$ Velocidad de sedimentación calculada mediante la ley de Stokes, tomando una temperatura del agua de $15^{\circ} \mathrm{C}$ 2 Índice y grado de dispersión según Folk and Ward (1957)

Las texturas del sedimento transportado durante las actuaciones en el cauce fueron ligeramente superiores a las texturas promedio obtenidas durante crecidas (p.ej. en una crecida de Junio 2015 el $\mathrm{D}_{50 \text {-medio }}=13 \mu \mathrm{m}$; Nogales, 2016; Fig. 5). Puntualmente, en la sección más próxima a la extracción, el tamaño medio transportado llegó a ser un orden de magnitud mayor que la media en crecidas $\left(D_{50 \text {-máximo }}=134 \mu \mathrm{m}\right)$. El 80\% de las partículas en suspensión muestreadas durante las actuaciones pertenecen a fracciones limosas. Este porcentaje es similar al obtenido para las partículas transportadas en suspensión durante crecidas naturales (Fig. 5). No obstante, es importante destacar que en la sección DS1 durante el día 18 el sedimento muestreado era más grueso, de modo que la fracción limosa solo llegó a representar el 64\% de la muestra. Julien (1998) evalúa el modo de transporte de sedimento en función del ratio entre la velocidad 
de sedimentación (Tabla 2) y la velocidad de corte $(0,176 \mathrm{~m} / \mathrm{s}$, para los parámetros estimados en los modelos hidráulicos (pendiente media $=0,007 \mathrm{~m} / \mathrm{m}$ y profundidad media $=0,4 \mathrm{~m})$. Según el rango de partículas observado $\left(\mathrm{D}_{50}=13-134 \mu \mathrm{m}\right)$, todas las partículas se transportarían en suspensión. Sin embargo las partículas de mayor tamaño quedan muy cerca del límite de transporte mixto. Estos resultados indican que las actuaciones en el cauce causaron la movilización de sedimentos de mayores tamaños que en condiciones naturales pero, como se ha indicado, estos materiales transportados en suspensión fueron sedimentados en los primeros metros aguas abajo debido a la ausencia de caudales competentes.

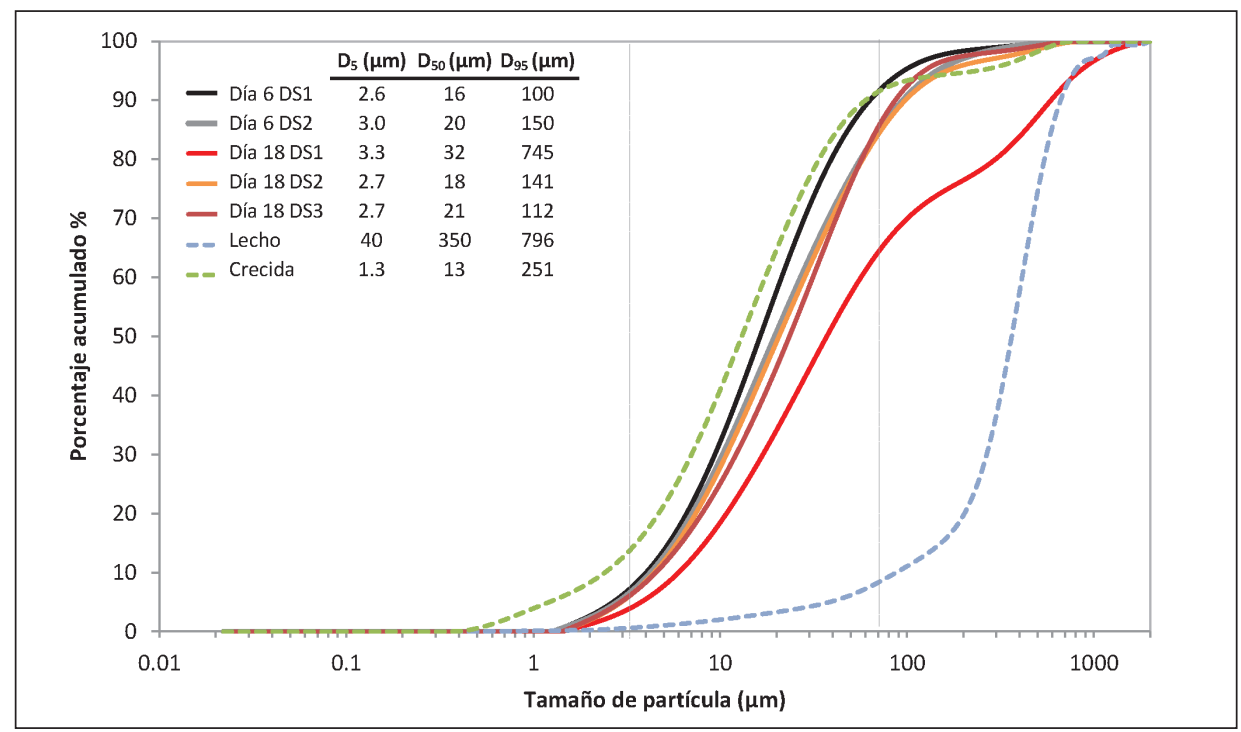

Figura 5. Distribución del tamaño de partículas en suspensión durante los trabajos asociados a la extracción de áridos en un tramo del alto Cinca en agosto 2014. Cabe destacar que se combinaron los datos de todas las muestras pertenecientes a la misma sección al mismo día para obtener las curvas. Las líneas de puntos muestran como referencia la distribución granulométrica de: (en azul) los parches de sedimento fino del lecho analizados, (en verde) el sedimento fino en suspensión en una crecida en Junio 2015 muestreada en la sección DS4 (Nogales, 2016). Las líneas de puntos verticales delimitan las distintas fracciones en la escala Wentworth (arcilla $<3,9 \mu \mathrm{m}$, limo $<63 \mu \mathrm{m}$, arena $<2000 \mu \mathrm{m}$ ). En la tabla se muestran los percentiles característicos de cada distribución granulométrica.

Es interesante destacar que el sedimento movilizado durante los días de la actuación es, en general, de menor tamaño que el material de los parches de finos del lecho (Fig. 5), aunque puntualmente, los tamaños transportados llegan a alcanzar valores similares a los del lecho (i.e. $\mathrm{D}_{95 \text {-lecho }}=796 \mu \mathrm{m} ; \mathrm{D}_{95 \text {-transportado }}=745 \mu \mathrm{m}$ durante el día 18 en DS1; Fig. 5). Estas fracciones de mayor tamaño representan materiales que no son transportados frecuentemente. Por tanto, los trabajos en el cauce ocasionan puntualmente el transporte de materiales de mayor tamaño procedentes del lecho que no son transportados frecuentemente durante crecidas, donde los sedimentos transportados en suspensión son más finos y proceden mayoritariamente de la 
cuenca. Tanto la sedimentación selectiva aguas abajo como la movilización de materiales de mayor tamaño, aunque afecte sólo a pequeños tramos del cauce, altera la porosidad de lecho y puede causar modificaciones en el hábitat, afectando a las comunidades bentónicas (Lisle, 1989; Buendía et al., 2011; Jones et al., 2012).

\section{Consideraciones finales}

Este artículo analiza los efectos de una extracción de áridos realizada en el marco de una actuación en el cauce para prevenir inundaciones sobre el transporte del sedimento en suspensión en el río Cinca. Las concentraciones medias observadas durante los días de estudio fueron mayores que en la sección de referencia aguas arriba de la actuación. Los resultados muestran cómo la actuación en el cauce alteró la dinámica del sedimento, sobre todo en el tramo situado inmediatamente aguas abajo. Los efectos de las actuaciones en el cauce sobre las concentraciones de sedimento y la carga sólida del río ya no se observaban a 1,5 km aguas abajo. La máxima concentración de sedimentos fue similar a las obtenidas en crecidas naturales; por su parte, la carga sólida total transportada como consecuencia de los trabajos en el cauce fue notablemente más baja que en crecidas. Finalmente, cabe señalar que el tamaño de partícula movilizado por la extracción fue similar o puntualmente mayor que la media transportada durante crecidas.

Los resultados muestran que el incremento de la carga sedimentaria debido a los trabajos en el cauce se asemeja a la que se produce durante las crecidas naturales. Este aumento repentino de las concentraciones, sin alteraciones sobre la hidráulica, conlleva efectos sobre la biota y el hábitat fluvial (Weeks et al., 2003). En este sentido, este trabajo muestra la alteración en la duración de la exposición a altas concentraciones, así como la alteración puntual en el tamaño de partícula transportada. Ambos factores son importantes ya que tienen efectos sobre la biota acuática (p.ej. deterioro de órganos respiratorios, abrasión, deriva involuntaria; Bilotta y Brazier, 2008; Béjar et al., 2017). A largo plazo las extracciones de áridos tienen también efectos en el balance sedimentario del tramo fluvial donde se localizan. Las actuaciones en el cauce suelen ejecutarse en época de caudales bajos para facilitar el acceso de las máquinas y aumentar la disponibilidad de material (i.e. cantidad de sedimento expuesto). Estos caudales no son competentes para transportar el sedimento que se moviliza en los trabajos de preparación del área y durante la extracción del material. Consecuentemente, el sedimento movilizado por la extracción se acumula en los primeros metros quedando disponible para crecidas posteriores. Los ríos de montaña no alterados por presas u otros impactos tienen ciclos naturales de sedimento (i.e. alternancia de periodos con cargas más altas y bajas en relación a los procesos de producción, almacenaje y transferencia de materiales en la cuenca). En estos ríos se alternan épocas de producción de sedimento en áreas fuentes (p.ej. meteorización de roca madre por procesos de hielo-deshielo en invierno y primavera), con periodos de transferencia del material al cauce fluvial por las lluvias y el deshielo en primavera y, finalmente, exportación del mismo por crecidas estivales y otoñales. Estos ciclos han estado descritos para cuencas vecinas a las del Cinca en los trabajos de López-Tarazón et al. (2011), Piqué et al. (2014), Lobera et al. (2016b). Así, la generación y redistribución de sedimentos producida por las actuaciones en el cauce puede alterar este ciclo natural 
y algunas funciones ecosistémicas asociadas (como por ejemplo la freza de los peces o la deriva de invertebrados) en función de la época del año en la que se realicen.

Se ha demostrado que los efectos de las extracciones de áridos sostenidas en el tiempo pueden propagarse varios kilómetros aguas abajo (Kondolf, 1994), e incluso aguas arriba (erosión remontante). Por ejemplo, en cuencas donde la transferencia de sedimentos se interrumpe por azudes y presas, el sedimento movilizado por la extracción quedará retenido en estas estructuras, contribuyendo a reducir su vida útil (Gilbert, 1917; Kondolf, 1997). Este estudio presenta un análisis cuantitativo no habitual en estudios sobre impactos fluviales, y supone un primer paso para la comprensión de la dinámica del sedimento en ríos localmente afectados por actuaciones en el cauce, y su influencia en la dinámica morfosedimentaria y la integridad ecológica del río.

\section{Agradecimientos}

Este trabajo se ha desarrollado en el marco del proyecto MorphSed (CGL201236394, www.morphsed.es) financiado por el Ministerio de Economía y Competitividad y el Fondo Europeo de Desarrollo Regional (FEDER). El primer autor tiene una beca predoctoral de la Universidad de Lleida. El segundo autor está contratado mediante el programa Ramón y Cajal (RYC-2010-06264) del Programa Nacional de Contratación e Incorporación de Recursos Humanos de Investigación del Ministerio de Economía y Competitividad. El Grupo de Investigación de Dinámica Fluvial-RIUS es Grupo Consolidado reconocido por la Generalitat de Catalunya (RIUS 2017 SGR 459). De la misma forma, se agradece al CERCA Programme de la Generalitat de Catalunya. Los datos hidrológicos han sido suministrados por la Confederación Hidrográfica del Ebro (CHE), mientras que los datos de caudal de los hidropicos han sido facilitados por Acciona. Los autores agradecen la participación de distintos miembros de RIUS en el trabajo de campo y las facilidades recibidas por el grupo de Hidrología Superficial y Erosión del Consejo Superior de Investigaciones Científicas para la obtención de las granulometrías de los sedimentos. Por último, este trabajo no hubiese sido posible sin la activa colaboración y participación de parte de la Confederación Hidrográfica del Ebro y de la empresa Horpisa, encargada de realizar las actuaciones en el cauce.

\section{Referencias}

Béjar, M., Gibbins, C.N., Vericat, D., Batalla, R.J. 2017. Effects of suspended sediment transport on invertebrate drift. River Research and Applications (en prensa). http://doi.org/10.1002/ rra.3146.

Bilotta, G.S., Brazier, R.E. 2008. Understanding the effects of suspended solids on water quality and aquatic biota. Water Research 42, 2849-2861. http://doi.org/10.1016/j.watres.2008.03.018.

Bladé, E., Cea, L., Corestein, G., Escolano, E., Puertas, J., Vázquez-Cendón, M.E., Dolz, J., Coll, A. 2014. Iber-Herramienta de simulación numérica del flujo en ríos. Revista Internacional de Métodos Numéricos 30, 1-10. http://doi.org/10.1016/j.rimni.2012.07.004.

Brown, A.V., Lyttle, M.M., Brown, K.B. 1998. Impacts of gravel mining on gravel bed streams. Transactions of the American Fisheries Society 127, 979-994. http://doi.org/10.1577/15488659(1998) $127<0979:$ IOGMOG>2.0.CO;2. 
Bryce, M.N. 1977. A study of sediment movements downstream from a hydraulic dredge. Unpublished Bachelor of Arts thesis, University of New South Wales.

Buendía, C., Gibbins, C.N., Vericat, D., López-Tarazón, J.A., Batalla, R.J. 2011. Influence of naturally high fine sediment loads on aquatic insect larvae in a montane river. Scottish Geographical Journal 127, 315-334. http://doi.org/10.1080/14702541.2012.670006.

Folk, R.S., Ward, W.C. 1957. Brazos river bar: a study in the significance of grain size parameters. Journal of Sedimentary Petrology 27, 3-26. http://doi.org/10.1306/74D70646-2B21-11D78648000102C1865D.

Forshage, A., Carter, N.E. 1973. Effect of gravel dredging on the Brazos River. Proceedings of the 27th Annual Conference, Southeastern Association Game and Fish Commission 24, 695-708. http://doi.org/10.1080/02705060.2008.9664250.

García-Anquela, J.A., Tena, J.M., Mandado, J.A. 1985. Las explotaciones de áridos como factor modificador de los cauces fluviales naturales. Cuadernos de Investigación Geográfica 11, 83-90. http://doi.org/10.18172/cig.945.

Gilbert, G.K. 1917. Hydraulic Mining Debris in the Sierra Nevada. US Geological Survey Professional Paper, 105.

Julien, P.Y. 1998. Erosion and sedimentation. Cambridge, Cambridge University Press, 280 pp.

Jones, J.I., Murphy, J.F., Collins, A.L., Sear, D.A., Naden, P.S., Armitage, P.D. 2012. The impact of fine sediment on macro-invertebrates. River Research and Applications 28, 1055-1071. http://doi.org/10.1002/rra.1516.

Kondolf, G.M. 1994. Geomorphic and environmental effects of instream gravel mining. Landscape and Urban Planning 28, 225-243. http://doi.org/10.1016/0169-2046(94)90010-8.

Kondolf, G.M. 1997. Hungry Water: Effects of Dams and Gravel Mining on River Channels. Environmental Management 21 (4), 533-551. http://doi.org/10.1007/s002679900048.

Lagasse, P.F., Winkley, B.R., Simons, D.B. 1980. Impact of gravel mining and river system stability. Journal of the Waterways, Port, Coastal and Ocean Division 106 (3), 389-404.

Lisle, T.E. 1989. Sediment transport and resulting deposition in spawning gravels, north Coastal California. Water Resources Research 25, 1303-1319. http://doi.org/10.1029/ WR025i006p01303.

Llena, M., Vericat D., Martínez-Casasnovas, J.A. 2016. Cambios geomorfológicos en el Alto Cinca (Periodo 1927-2014). Comprendiendo el relieve: del pasado al futuro. En: J.J. Durán Valsero, M. Montes Santiago, A. Robador Moreno, A. Salazar Rincón, Actas de la XIV Reunión Nacional de Geomorfología, Málaga, 2016, IGME: Madrid, pp. 339-347.

Lobera, G., Muñoz, I., López-Tarazón, J.A., Vericat, D., Ramon, R.J. 2016a. Effects of flow regulation on river bed dynamics and invertebrate communities in a Mediterranean River. Hydrobiologia 784, 283-304. http://doi.org/10.1007/s10750-016-2884-6.

Lobera, G., Batalla, R.J., Vericat, D., López-Tarazón, J.A., Tena, A. 2016b. Sediment transport in two Mediterranean regulated rivers. Science of The Total Environment 540, 101-113. http:// doi.org/10.1016/j.scitotenv.2015.08.018.

López-Tarazón, J.A., Batalla, R.J., Vericat, D. 2011. In-channel sediment storage in a highly erodible catchment: the River Isábena (Ebro basin, Southern Pyrenees). Zeitschrift für Geomorphologie 55 (3), 365-382. http://doi.org/10.1127/0372-8854/2011/0045.

Mori, N., Brancelj, A. 2011. Invertebrate drift during in-stream gravel extraction in the River Bača, Slovenia. Fundamental and Applied Limnology 178 (2), 121-130. http://doi. org/10.1127/1863-9135/2011/0178-0121.

Newcombe, C.P., Macdonald, D.D. 1991. Effects of Suspended Sediments on Aquatic Ecosystems. North American Journal of Fisheries Management 11 (1), 72-82. http://doi.org/10.1577/15488675(1991)011<0072:EOSSOA>2.3.CO;2. 
Nogales, I. 2016. Dinámica del transporte de sedimentos en suspensión en un río de montaña afectado por extracciones de áridos (Alto Cinca, Pirineo Central). Trabajo Fin de Máster. Universidad de Lleida.

Parkhill, K.L., Gulliver, J.S. 2002. Effect of inorganic sediment on whole-stream productivity. Hydrobiologia 472, 5-17. http://doi.org/10.1023/A:1016363228389.

Piqué, G., López-Tarazón, J.A., Batalla, R.J. 2014. Variability of in-channel sediment storage in a river draining highly erodible areas (the Isábena, Ebro Basin). Journal of Soils and Sediments 14, 2031. http://doi.org/10.1007/s11368-014-0957-6.

Pretty, J.L., Hildrew, A.G., Trimmer, M. 2006. Nutrient dynamics in relation to surface-subsurface hydrological exchange in a groundwater fed chalk stream. Journal of Hydrology 330, 84-100. http://doi.org/10.1016/j.jhydrol.2006.04.013.

Rinaldi, M., Wyżga, B., Surian, N. 2005. Sediment mining in alluvial channels: physical effects and management perspectives. River Research and Applications 21, 805-828. http://doi. org/10.1002/rra.884.

Rovira, A., Batalla, R.J., Sala, M., 2005. Response of a river sediment budget after historical gravel mining (the lower Tordera, NE Spain). River Research and Applications 21 (7), 829-847. http://doi.org/10.1002/rra.885.

Vericat, D., Batalla, R.J. 2006. Sediment transport in a large impounded river: the lower Ebro, NE Iberian Peninsula.Geomorphology 79,72-92.http://doi.org/10.1016/j.geomorph.2005.09.017

Vericat, D., Muñoz-Narciso, E., Béjar, M., Ramos-Madrona, E. 2016. Case study: Multi-temporal reach-scale topographic models in a wandering river -uncertainties and opportunities. En: J.L. Carrivick, M.W. Smith, D.J. Quincey (Eds.), Structure from Motion in the Geosciences. New Analytical Methods in Earth and Environmental Sciences, Wiley Blackwell, John Wiley \& Sons, Chichester, UK, pp 194.

Waters, T.F. 1995. Sediment in streams Sources, biological effects, and control. American Fisheries Society Monograph 7, 1-251.

Warner, R.F., McLean, E.J., Pickup G. 1977. Changes in an urban water resource, an example from Sydney, Australia. Earth Surface Processes 2, 29-38. http://doi.org/10.1002/esp.3290020104.

Weeks, J.M., Sims, I., Lawson, C., Harrison, DJ. 2003. River mining: assessment of the ecological effects of river mining in the Rio Minho and Yallahs rivers, Jamaica. Brithish Geological Survey Commissioned Report 53.

Williams, G.P., Wolman, M.G. 1984. Downstream effects of dams on alluvial rivers. US Geological Survey Professional Paper 1286, 83. http://doi.org/10.1002/rrr.3450010210.

Wood, P.J., Armitage, P.D. 1997. Biological effects of fine sediment in the Lotic environment. Environmental Management 21, 203-217. http://doi.org/10.1007/s002679900019. 